\title{
Prácticas restaurativas en el marco jurídico mexicano con enfoque a una cultura de paz
}

\author{
Restorative practices in the mexican juridical framework with a focus on a Culture of peace. \\ Práticas restaurativas no marco jurídico mexicano com enfoque a uma cultura de paz.
}

DOI: http://dx.doi.org/10.21803\%2Fpenamer.11.21.526

Yahaira Berenice Martínez Pérez ORCID 0000-0002-4047-7298

Gabriel de Jesús Gorjón Gómez ORCID 0000-0001-5033-9377

\section{¿Cómo citar este artículo?}

Martínez, Y. \& Gorjón, G. (2018). Prácticas restaurativas en el marco jurídico mexicano con enfoque a una cultura de paz. Pensamiento Americano, 11(21), 79-95.

DOI:http://dx.doi.org/10.21803\%2Fpenamer.11.21.526

\begin{abstract}
Resumen
El presente artículo deviene de un proyecto de investigación socio jurídico con aplicación al derecho comparado, es de tipo reflexivo y analítico, de tal forma se ha dividido en dos etapas: la primera que atribuye el marco teórico y la segunda busca instrumentalizar los procesos restaurativos en México. Ahora bien, se pretende demostrar la importancia del uso de la Justicia Restaurativa en su nivel trasformativo y sanador al delito, en virtud de que los programas restaurativos tales como: Encuentros restaurativos, conferencias restaurativas y Círculos de Paz buscan promover un clima positivo en el comportamiento humano, generando una cultura de paz en la sociedad. Lo anterior de un abordaje cualitativo, a través un método descriptivo, con el objetivo de un análisis comparativo en la fenomenología de estudio. En este sentido, se encontró que los diversos programas de justicia restaurativa fomentan valores universales en función a la No violencia y la cultura del diálogo para efectos de solucionar conflictos de índole penal dentro de un ambiente cordial en las comunidades, así mismo, de manera colateral funge como disuasivo de la conducta lesiva.
\end{abstract}

PALABRAS CLAVE: Mecanismos alternativos de solución de controversias, Encuentros restaurativos, Conferencias restaurativas, Círculos de Paz, Cultura de Paz.

\begin{abstract}
The present article comes from a socio-legal research project with application to comparative law, it is of a reflective and analytical type, so it has been divided into two stages: the first one that attributes the theoretical framework and the second seeks to instrumentalize the restorative processes in Mexico. However, it is intended to demonstrate the importance of the use of Restorative Justice in its transformative and healing level to crime, by virtue of the fact that restorative programs such as: Restorative meetings, restorative conferences and Circles of Peace seek to promote a positive climate in behavior human, generating a culture of peace in society. The previous of a qualitative approach, through a descriptive method, with the objective of a comparative analysis in the study phenomenology. In this sense, it was found that the various programs of restorative justice promote universal values based on nonviolence and the culture of dialogue for the purpose of solving conflicts of a criminal nature within a cordial environment in the communities, likewise, in a collateral manner. serves as a deterrent to the harmful behavior.
\end{abstract}

KEYWORDS: Alternative dispute resolution mechanisms, Restorative meetings, Restorative conferences, Circles of Peace, Culture of Peace.

Resumo 
O presente artigo se origina de um projeto de pesquisa sócio-jurídico com aplicação al direito comparado, é de tipo reflexivo e analítico, de tal forma que se dividiu em duas etapas: a primeira que se atribui ao marco teórico e a segunda que busca instrumentalizar os processos restaurativos no México. Agora bem, pretende-se demonstrar a importância do uso da Justiça Restaurativa no seu nível transformador e sanador do delito, em virtude de que os programas restaurativos tais como: os Encontros restaurativos, as conferências restaurativas e os Círculos de Paz buscam promover um clima positivo no comportamento humano, gerando uma cultura de paz na sociedade. O anterior de uma abordagem qualitativa, através de um método descritivo, com o objetivo de uma análise comparativa na fenomenologia do estudo. Neste sentido, se encontrou que os diversos programas de justiça restaurativa fomentam valores universais em função à Não violência e a cultura do diálogo para efeitos de solucionar conflitos de índole penal dentro de um ambiente cordial nas comunidades, assim mesmo, de maneira colateral aparece como dissuasivo da conduta lesiva.

PALAVRAS CHAVE: Mecanismos alternativos de solução de controvérsias, Encontros restaurativos, Conferências restaurativas, Círculos de Paz, Cultura de Paz.

\section{Perfil}

Doctora en Métodos Alternos y Solución de Conflictos, Maestra en Métodos Alternos y Solución de Conflictos por la Facultad de Derecho y Criminología de la Universidad Autónoma de Nuevo León. Docente de la UANL, Docente en postgrado en el Instituto de la Judicatura Federal, Facilitador con Certificación Nacional.

E-mail: yahairamtz@hotmail.com

\section{Perfil}

80 Doctor en Derecho por la Universidad Autonoma de Nuevo Leon; Maestría en Derecho Corporativo; Facilitador certificado; Miembro de la Asociacion Internacional de Doctores en MASC. Profesor de Tiempo Completo de la Universidad Autonoma de Nuevo Leon. Miembro del Sistema Nacional de Investigadores del CONACyT Nivel I, Delegado de la Confederacion de Colegios y Asociaciones de Abogados de Mexico A.C. CONCAAM.gorjon_g_g@hotmail.com; ggorjon@hotmail.com

Yahaira Martínez Pérez Licenciada en Derecho y Ciencias Sociales.

Gabriel Gorjón Gómez

Licenciado en Derecho 


\section{Introducción}

E presente artículo deriva del estudio de un proyecto de investigación de tesis doctoral (Martínez, 2017), bajo el tema de Justicia Restaurativa en centros penitenciarios con la intención de abordar en particularidad los elementos que integran los procesos restaurativos en el sistema penal.

En este orden de ideas, en virtud de la reforma del 2008 se ha evidenciado el origen de la justicia restaurativa como una evolución transformativa en el derecho penal, toda vez que el Estado mexicano ha implementado dentro del ordenamiento legal los conceptos de Justicia Alternativa y Justicia Restaurativa, lo que ha llegado a confundirse respecto a su aplicación.

El sistema penal mexicano se había distinguido por un sistema de normas inquisitivas, ante la falta de modalidades de carácter preventivo y de reinserción social, lo que ha implicado que nuestra legislación de sistema judicial y de procuración de justicia estén acordes con los Instrumentos Internacionales y promuevan los derechos humanos y la reparación del daño con una nueva política garantista.

En virtud de lo anterior, surgió la idea de confeccionar dos vertientes en el área temática, mismas que se complementan con los objetivos de la investigación, en el primer punto se realizará un análisis del marco jurídico actual mexicano en relación con los Mecanismos Alternativos de solución de Controversias (MASC) y el segundo punto incluye el ejercicio de la Justicia Restaurativa.

La Justicia Restaurativa cambia su perspectiva en cuanto a la justicia tradicional en virtud de tomar como protagonista a la víctima, en esta formalidad ha resultado naturalmente interesante tener un acceso directo a la justicia con una visión humanista, transformando el crimen y las condiciones que lo propiciaron, buscando la relación directa del ofensor, así como de todos aquellos lesionados de su bien jurídico.

Si bien la Organización de las Naciones Unidas (2006), establece diversos programas de aplicación en ejercicio de la justicia restaurativa, el objetivo de la presente indagación es hacer un estudio de los siguientes programas:1) Encuentros restaurativos; 2) Conferencias restaurativas y 3) y el Círculo de Paz, esto con un análisis de aplicación en el derecho comparado.

\section{Metodología}

El artículo desarrolla una metodología cualitativa, se observa que integra característica de índole descriptivo con la intención de llevar a cabo un análisis comparativo, lo anterior con fundamento a diversa literatura nacional e internacional realizado a través de un proceso hermenéutico con la finalidad de comprender los textos en un sentido intencional y contextual (Cárcamo Vásquez, 2005). Lo anterior para dar una percepción asertiva de manera global y sustentada con el objetivo de aportar en la política pública (Hernández Sampieri, Fernández Collado, \& Baptista Lucio, 2006).

La revisión de distintos aspectos que atribuye la literatura del derecho comparado pone de manifiesto la necesidad de profundizar en la normativa jurídica nacional con especial interés de instrumentalizar los procesos restaurativos, de tal manera que el operador de justicia y las instituciones penitenciarias logren emprender un plan estratégico sanador para los participantes.

\section{Los MASC en el Estado Mexicano}

En las últimas décadas hemos venido observando que el sistema de justicia del Estado Mexicano ha evolucionado de manera positiva hasta convertirse en un sistema acusatorio y oral. En este orden de ideas, uno de los principales cambios del proceso incluye la aplicación de Mecanismos Alternativos de Solución de 
Controversias (MASC) en materia penal como respuesta a las necesidades de la sociedad moderna en un sistema más robustecido, estimulando los derechos humanos (Martínez, 2017).

Bajo este contexto, la Máxima Carta establece en el párrafo quinto del numeral décimo séptimo lo siguiente (Diario Oficial de la Federación, 2017):

"Las Leyes preverán mecanismos alternativos de solución de controversias. En materia penal regularán su aplicación, asegurarán la reparación del daño causado a la víctima y establecerá los casos en que se requerirá supervisión judicial".

Dicho precepto legal indica de manera primordial restituir lo dañado a las personas víctimas en el proceso penal con la aplicación de Mecanismos Alternativos de Solución de Controversias, donde ambas partes víctima y ofensor podrán llegar al consenso utilizando el diálogo a través del precepto pacta sunt servanda, la transparencia y la legalidad del proceso con miras a obtener una pronta respuesta en la justicia y satisfacer las necesidades de los participantes.

Por ende, algunos Estados de la República Mexicana iniciaron con reformas a sus respectivas Constituciones reconociendo en su mandato la justicia alternativa y justicia restaurativa. Tal es el caso de los estados de Aguascalientes, Baja California, Chihuahua, Coahuila, Colima, Distrito Federal, Guanajuato Hidalgo, Nuevo León, de Oaxaca, Quintana Roo, Sonora, Veracruz, Yucatán y Zacatecas, entre algunos por mencionar.

Ahora bien, los MASC se han reconocido por ser multidisciplinarios, bajo las características de autocompositivos pues las partes toman protagonismo en el proceso en la toma de sus propias decisiones mediante del diálogo, lo cual contribuye a resarcir el daño de una manera equitativa (Galain, 2009), se puede entender un mecanismo simple imparcial y trasparen- te (Gorjón, 2013), que se desarrolla mediante la normativa que el Estado establece a los operadores en la justicia (Eiras, 2005).

Así mismo, la mediación fortalece las relaciones personales en cuanto coadyuva a que los participantes logren asumir las consecuencias de los hechos, donde se observa la existencia de un acercamiento directo y por formalidad autónoma buscan solucionar al problema. En este contexto, la Justicia Alternativa tiene relevancia en distintos aspectos, en primer orden por la búsqueda de soluciones considerando el principio de autonomía de los participantes y por otro lado porque fortalece la Paz social (Blanco Suárez, 2014).

Ahora bien, la conciliación también es un mecanismo que se reconoce por la normativa de derecho para solucionar problemas, ante el contexto de llegar a un consenso con la intervención de un conciliador quien podrá dar propuestas ante la controversia (Ahumada, 2011). Esta personalidad quien funge como tercero imparcial en el proceso puede participar con la venia de los participantes cuya finalidad será formular las mejores propuestas en la resolución del conflicto.

El facilitador-conciliador debe dirigir el procedimiento bajo las características de un líder natural, imponerse con capacidad y habilidad de controlar el proceso en momentos de catarsis y sobre todo llevándolos a una finalidad en común, el acuerdo (Arboleda López, Gárces Giraldo, Murillo Bocanegra, \& Pineda Carreño, 2017).

La mediación penal también reconoce la intervención de una tercera persona, con la diferencia que esta figura de características imparcial y neutral con desarrollo de estrategias comunicativas no puede plantear propuesta alguna en la controversia; el facilitador-mediador se limita únicamente a guiarlos en el proceso penal de una manera equitativa bajo el contexto de plas- 
mar formalmente la lluvia de ideas de los participantes en el acuerdo reparatorio.

Lo anterior trae como consecuencia el desistimiento del acto delictivo lo cual favorece en la descarga laboral y celeridad del procedimiento que acontece en el sistema (Gorjón \& Steele, Metodos Alternativos de Solución de Conflictos, 2008). En este sentido, la aplicación de los métodos alternativos demuestra a las sociedades una forma sencilla flexible y pacífica para llegar a un consenso (Eiras, 2005), pues a través de los principios de voluntariedad, confidencialidad, neutralidad, imparcialidad, equidad y honestidad se contribuye a la cultura de paz en las comunidades.

\section{Estudio a la Ley Nacional de Mecanismos Al- ternativos de Solución de Controversias en Materia Penal (LNMASCMP).}

Como se ha venido comentando, la reforma al artículo 17 Constitucional evidencia de manera directa la respuesta a las controversias penales pues regula el ámbito de aplicación para las entidades federativas (Cámara de Diputados del H. Congreso de la Unión, 2014), de tal manera que favorece en la satisfacción de intereses de los particulares mediante una participación directa de la víctima el ofensor y la comunidad. En estos procesos puede observarse la oportunidad de solucionar el conflicto desde la etapa inicial del proceso.

La Ley en comento sustenta preceptos legales conducentes a la mediación, conciliación y justicia restaurativa y así mismo permite observar un primer panorama jurídico operativo en torno a los alcances y efectos que la implementación del sistema desarrolla actualmente en la sociedad mexicana (Martínez, 2017). Ante los principios de oralidad, información, transparencia, equidad, voluntariedad y confidencialidad, genera un parteaguas en la nueva administración de justicia lo que trae como consecuencia la despresurización judicial (Gorjón Gómez, y otros, 2015). Bajo este contexto, partiremos del estudio de prácticas restaurativas, entendiendo para tal efecto encuentros restaurativos, juntas restaurativas, conferencias y círculos de paz.

Ahora bien, la junta restaurativa se establece en el arábigo 27 de la Ley Nacional de Mecanismos Alternativos de Solución de Controversias en Materia Penal (Diario Oficial de la Federación, 2017), que a la letra reza:

"La junta restaurativa es el mecanismo mediante el cual la víctima u ofendido, el imputado $y$, en su caso, la comunidad afectada, en libre ejercicio de su autonomía, buscan, construyen y proponen opciones de solución a la controversia, con el objeto de lograr un acuerdo que atienda las necesidades y responsabilidades individuales y colectivas, así como la reintegración de la víctima $u$ ofendido y del imputado a la comunidad y la recomposición del tejido social".

Esta normativa apertura en el sistema penal mexicano la oportunidad que tiene el infractor de la norma de resarcir el daño mediante programas de índole restaurativo se busca llegar al punto más alto del lado humano concientizándole los efectos que trae consigo la conducta lesa y de manera colateral se le brinda la oportunidad de reintegrarse a la comunidad y reestructurar el tejido social que ha quebrantado.

En el mismo contexto se observa que la intervención de las sociedades funge en la mejora de las relaciones y de conducta de las personas, pues a través de la participación y el compromiso que obtienen las partes se adquiere una responsabilidad que va más allá de la cuestión pecuniaria, pues contribuye en robustecer los valores que integra la Paz Social (Cabello, 2015).

Cabe resaltar dos circunstancias elementales, de primera instancia la Ley Nacional de Mecanismos Alternativos de Solución de 
Controversias en Materia Penal (Diario Oficial de la Federación, 2017), se restringe en describir solamente la junta restaurativa empero los Tratados Internacionales reconocen una amplia gama de procesos restaurativos (Organización de las Naciones Unidas, 2006), mismos que sustenta el "Manual sobre programas de justicia restaurativa", tales como mediación entre víctima ofensor, conferencias restaurativas, sentencias de círculos y círculos de paz (Naciones Unidas, 2015), el ordenamiento jurídico local no preceptúa un marco normativo más consolidado en esta materia.

En este sentido nos encontramos ante la necesidad de especificar cada esquema restaurativo, en el desarrollo de su aplicación es fundamental que el intérprete logre identificar la metodología a seguir en cada uno de los procesos restaurativos y tener un panorama fortalecido de herramientas para el ejercicio de la Justicia Restaurativa (Martínez, 2017).

En segunda instancia la Ley citada al rubro (Diario Oficial de la Federación, 2017), establece los acuerdos en el capítulo VI, sin embargo se encuentra muy limitada ya que únicamente resalta los elementos necesarios para la conformación de este instrumento jurídico, en este sentido es necesario considerar las diferencias que resultan de la integración de un acuerdo reparatorio que se da en la mediación penal donde se faculta para su aprobación al Ministerio Público o al Juez de Control en la etapa inicial y del plan de reparación que se formula por el Ministerio Público o por el mismo imputado como respuesta al planteamiento que se observa en la etapa procesal referente a la suspensión condicional del proceso.

Cabe advertir la diferencia que resulta de un Acuerdo Restaurativo, donde el alcance legal sustenta la posibilidad de resultados restaurativosy por ende el elemento del perdón moral, lo cual no obstaculiza el ius puniendi de una pena privativa de libertad. Ahora bien, si las circunstancias favorecen a su excarcelación ya sea en cualquiera de sus modalidades de libertad condicionada, libertad anticipada, sustitución y suspensión temporal de las penas, permisos humanitarios o preliberación por criterios de política penitenciaria (Cámara de Diputados del H. Congreso de la Unión, 2016), se debe considerar que el ofensor podrá retornar a la vida en sociedad con otra visión de las cosas para mejorar su vida al reinsertarse a un nuevo modus vivendi (Martínez, 2017).

Cabe señalar un punto importante referente a la Ley Nacional de Ejecución Penal, dicho ordenamiento reconoce a la justicia restaurativa como parte del plan de actividades para reinsertar al individuo, mismo que se regula en sus ordinales 3 fracción $X X$, 203, 204, y que se rige por los principios de voluntariedad, flexibilidad, responsabilidad, confidencialidad, neutralidad, honestidad, es importante mencionar que el compendio legal suma un último ingrediente distinguiéndose ante los MASC, pues la normativa reconoce la reintegración del individuo.

\section{Análisis al Código Nacional de Procedimien- tos Penales.}

Dentro del marco constitutivo en el respeto a los derechos humanos reconocidos por la Carta Magna y en los Tratados Internacionales donde Estado mexicano forma parte, se establecen normas de investigación para el esclarecimiento de los delitos a través de una metodología jurídica impuesta por las directrices que el ordenamiento legal refiere en la procuración de la justicia (Martínez, 2017).

Los Mecanismos Alternativos de Solución de Controversias se encuentran presentes en diversos momentos del proceso penal, la mediación y conciliación se pueden observar en la primera etapa del procedimiento establecido por el Código Nacional de Procedimientos 
Penales (CNPP), dichos procesos son guiados por un facilitador quien tiene figura de líder y el cual desarrolla diversas técnicas de comunicación, con habilidades y destrezas en el manejo de emociones para motivar a los particulares a formular acuerdos (Arboleda López, Gárces Giraldo, Murillo Bocanegra, \& Pineda Carreño, 2017), lo cual permite liberar la enorme carga de trabajo que origina la comisión del delito de menor cuantía.

García Ramírez (2014), reconoce la mediación penal y la conciliación como mecanismos alternativos de controversias y así mismo el artículo 184 del CNPP manifiesta en su precepto legal como salidas alternas el acuerdo reparatorio y la suspensión condicional del proceso (Diario Oficial de la Federación, 2017), se confirma que solo procederán aquellos acuerdos que sean aprobados por el Ministerio Público o el Juez de control bajo el ordinal 187 CNPP, por lo tanto, el criterio de oportunidad aplica en los delitos que se persiguen de querella o que admiten el perdón de la víctima u ofendido; delitos culposos y delitos patrimoniales cometidos sin violencia, eximiendo los delitos de violencia familiar o cuando el imputado haya firmado con anterioridad otros acuerdos por el mismo delito doloso salvo que haya cumplido en una periodicidad de dos años.

Otros autores se refieren a los acuerdos como negocios jurídicos procesales (Carnelutti, 1982), en virtud de adquirir efecto legal a través de la aprobación del órgano competente del proceso penal, tal como se ha venido comentando en párrafos anteriores dichos procesos se rigen por los principios de autonomía, flexibilidad, celeridad del proceso, la economía procesal y la buena fe de las partes.

Cabe mencionar la intervención del Ministerio Público y Juez de Control como figuras facultadas para autorizar el acuerdo considerando que cumpla con el principio de legalidad y proporcionalidad, es decir, bajo que hipótesis se sustenta el principio de autocomposición en caso de no aprobarse el convenio y quedar sin efectos jurídico, por consiguiente se puede advertir una postura contradictoria, ante estas circunstancias cabría cuestionar el principio de voluntariedad al que se sometieron los participantes.

\section{Ley General de Víctimas. Contexto General.}

El estado de transición que se ha venido experimentando en la primera década del año 2000 con aplicación al nuevo sistema penal acusatorio enfoca una evolución en los Derechos humanos en la República Mexicana, aunado a ello se han originado diversos movimientos sociales que han alertado a la Nación en la necesidad de protección a la ciudadanía.

De primera instancia la Carta Magna establece en el artículo 20 un conjunto de derechos hacia la víctima en atención a los procedimientos penales, tales como recibir atención médica y psicológica, recibir asesoría jurídica, el resguardo de la identidad, solicitar medidas cautelares y providencias necesarias para garantizar su seguridad, así como la reparación del daño e impugnar las omisiones del ministerio Público en el proceso penal, entre algunas otras por mencionar.

Ahora bien, La Ley General de Víctimas tiene su origen en el Estado Mexicano en fecha 9 de enero del 2013, bajo el artículo 2 denota los derechos advertidos en la Constitución, y así mismo reconoce la protección de los derechos humanos, garantiza las reparaciones integrales y de la misma manera establece el derecho a una justicia pronta y expedita hacia las víctimas. Bajo este contexto, la víctima adquiere especial atención, pues desde un punto de vista de la victimología únicamente se había considerado al delincuente haciendo de lado a quien sufrió la lesión del daño (Neuman, 2005).

Siguiendo ese mismo orden de ideas, Rousset (2011) establece que la reparación debe cumplir 
con una modalidad de elementos requeridos por el Sistema Interamericano de Derechos Humanos. Dicho protocolo reconoce las medidas cautelares, con el objetivo de evitar daños irreparables en las víctimas, establece también el derecho a las peticiones individuales, tal es el caso de la solicitud hecha en fecha 23 de Febrero del 2008 a la Corte Interamericana de Derechos humanos por representantes de las víctimas que integran el caso Campo Algodonero, con el objetivo de realizar investigaciones eficaces en los procesos penales y que trajo como consecuencia la resolución de la sentencia 2009/01/19 como respuesta a la violencia de género.

Así mismo, reconoce la aplicación de medidas alternas donde previo consentimiento de las partes se busca la resolución de conflictos con la finalidad de enmendar los daños y terminar con el proceso penal. Cabe mencionar Tesis de Jurisprudencia 145/2005 relacionada con reparación del daño (Suprema Corte de Justicia de la Nacion, 2006), de tal forma que asegura de manera puntual y suficiente la protección a sus derechos fundamentales y responde al reclamo social frente a la impunidad y a los efectos del delito sobre ciudadanos (Martínez, 2017).

Bajo este mismo contexto, se ha legitimado que la víctima tenga derecho a la reparación de daño económico y psicológico con la finalidad de lograr así una clara y plena reivindicación de dichos efectos en el proceso penal; en este sentido la reparación del daño tiene el carácter de pena pública y por ende al ser parte de la condena impuesta en el procedimiento penal deberá confirmarse la pena pública desde el momento que se acredita el delito mediante responsabilidad activa del infractor para restablecer el daño que originó a la víctima (Suprema Corte de Justicia de la Nacion, 2006).

Cabe mencionar que algunos países que practican la Justicia Restaurativa concretan en sus preceptos legales el beneficio a todos los in- tegrantes en la controversia penal, esto es, no solamente buscan el beneficio para la víctima sino también integran al ofensor y las comunidades que se ven afectadas por el delito, con el objetivo de restaurar los tejidos sociales y contribuir en el cumplimiento de una Paz Social.

\section{Organización de las Naciones Unidas.}

El papel que ha venido desempeñando las $\mathrm{Na}-$ ciones Unidas entorno a la justicia alternativa ha inspirado a diversos países en la implementación de los mecanismos alternativos de solución de conflictos reconociendo como tal a la mediación, conciliación y la justicia restaurativa.

Los primeros antecedentes que refieren los conceptos de mediacón y justicia restaurativa se fundamentan por el Consejo Económico y Social en la Resolución 2000/14 donde establece: "Principios Básicos sobre Utilización de Programas de Justicia Restaurativa en Materia Penal" (Organización de las Naciones Unidas, 2006), se hace alusión al respeto de los derechos humanos reconocidos en los tratados universales, así como el principio de autonomía que tienen los participantes para su intervención teniendo en consideración la participación activa de las comunidades.

Por lo tanto, con fundamento al derecho comparado y a las directrices establecidas por los instrumentos internacionales se debe considerar de suma importancia implementar programas pacíficos de resolución de controversias con metodología colaborativa efectiva en materia de transformación del acto humano en un amplio entendimiento y comprensión respecto a la conducta delictiva con miras a un resultado positivo para los protagonistas del conflicto (Martínez, 2017).

Siguiendo este orden de ideas, la Declaración de Viena celebrada en el año 2000 por la Asamblea de las Naciones Unidas describe los convenios a los que se someten los países integrantes 
donde establece la universalidad de los derechos humanos y se observan modelos de colaboración cuyo objetivo es la no reincidencia del delito y la justicia penal. Es cinco años más tarde cuando las Naciones Unidas integran procedimientos y programas de justicia restaurativa estableciendo para tal efecto en su décimo primer Congreso sobre la Prevención del Delito y Justicia Penal Celebrado en Bangkok (Organización Naciones Unidas, 2014).

Dicho contexto expone: "La declaración sobre los principios fundamentales de justicia para las víctimas" (Organización de las Naciones Unidas, 2006), en éste reconoce el derecho a las víctimas a recibir un trato justo e instituye la oportunidad a desarrollar un mecanismo alternativo de solución de controversias, con la finalidad de facilitar el acceso a la justicia para que en el menor tiempo posible sea reparado el daño.

Ahora bien, para dar inicio en un proceso de mediación víctima-delincuente se deben considerar diversos aspectos a seguir a través del "Manual sobre programas de Justicia Restaurativa" (Naciones Unidas, 2015):

a) La aceptación del delincuente de su responsabilidad.

b) La participación de la víctima y el delincuente.

c) Los participantes deben considerar si es seguro participar en el proceso.

El referido manual se integra por diversas secciones, refiere conceptos de justicia restaurativa, establece los diferentes programas de aplicación, así como las modalidades de intervención para obtener un proceso de restauración exitoso. Así mismo fundamenta los parámetros a seguir para garantizar los derechos a los participantes en los procesos restaurativos, tales como el derecho a la información, el derecho a consultar un abogado, el derecho al menor de estar representado por su padre madre o tutor, así como el dere- cho a elegir si quiere participar en el proceso.

Es relevante mencionar que los procesos restaurativos impulsan una cultura de diálogo, lo cual trae consigo una cultura de Paz en las sociedades (Neuman, 2005), bajo este contexto, es necesario promover diversos valores como el bienestar, la igualdad, la equidad, la identidad, la seguridad, contemplando modificar conductas sin necesidad de recurrir a la violencia (Islas Colín, Vera H., \& Miranda-Medina, 2018).

De tal manera, con ello se busca direccionar a los participantes en un punto de vista más humano, es decir que el ofensor enmiende los daños que ha causado y así logre a sumir las consecuencias de sus actos y de manera colateral prevenir la reincidencia del delito.

\section{Actuación de la Justicia Restaurativa}

La justicia restaurativa también conocida en algunos países como justicia restitutiva o justicia reparadora, para el Estado Mexicano ha representado un cambio meramente trasformativo en el derecho penal, se ha evidenciado con su implementación la evolución de un sistema retributivo a un sistema humanizado de justicia. Se reconoce que la aplicación de los programas de justicia restaurativa proporciona sanciones más constructivas además de evitar el congestionamiento administrativo que acontece el sistema de justicia penal.

Siguiendo este orden de ideas, la justicia restaurativa considera necesario la participación de diferentes figuras, siendo éstos el facilitador, la víctima, el ofensor y la comunidad, para ello es necesario desarrollar estrategias de comunicación donde el ofensor asume las consecuencias de la conducta atípica y ofrece su disposición en reparar la transgresión a la norma. De tal manera, prepondera solucionar el conflicto de una forma positiva dejando atrás el castigo que acontece en el sistema represivo (Olson, 2001). 
Versus la aplicación de la norma punitiva que incorpora la pena privativa de la libertad, como respuesta al ius punendi del Estado, en este sentido, en lo que atribuye al delito se ha evidenciado su efecto fallido en consideración a la reintegración social (Mosquera Rentería, 2015). Ahora bien, la ciencia del derecho penal fundamenta la sanción penal como mecanismo de restitución de la convivencia social que ha sido alterada (Carrillo Pumarejo \& Álvarez Martínez, 2012). Bajo este contexto, el crimen afecta directamente a las personas de manera emocional y económica y así mismo a la comunidad entorno al margen social. De tal forma, habría que profundizar en el tema de justicia restaurativa y dar respuesta a la comisión del ilícito.

Howard Zehr establece la posibilidad al ofensor de la norma de relatar las causas del delito, por lo tanto apertura la reparación del daño (Zerh, 2007), es decir, la instauración de programas de índole restaurativo protagoniza a la víctima $y$ al ofensor con efectos transformadores en protección a los intereses del pasivo, con cuidado a la no revictimización robusteciendo los grupos de apoyo en las comunidades.

La Organización de las Naciones Unidas (ONU) refiere una gamma metodológica de contenido restaurativo para aplicar en los procesos penales, entre ellos la mediación entre víctima y delincuente, conferencias en grupos, sentencias en círculos, libertad condicional y juntas restaurativas. Cabe hacer mención que la presente investigación considera hacer un estudio conforme al Derecho comparado en los siguientes programas: 1) Encuentros restaurativos; 2) Conferencias restaurativas; y 3) el Círculo de Paz.

\section{Encuentros restaurativos}

Se ha evidenciado que la coercitividad aplicada por el Estado conforme al endurecimiento de penas no ha sido benévola en la disuasión del delito (Barnett, 1977). Esto es, al referir el sistema punitivo orientamos de primer orden el castigo al ejecutor del delito, por ende, la aplicación a una norma privativa de la libertad en reclusión. Bajo este contexto se debe considerar aplicar estrategias restaurativas que permitan a los participantes trasformar sus vidas en un sentido positivo, pues se ha confirmado que el desarrollo de estas prácticas coadyuva en la prevención de las conductas atípicas (Umbreit \& Cores, 2006).

Ahora bien, la mayor parte de los países que aplican modelos restaurativos denominan el encuentro restaurativo como mediación entre víctima y ofensor, mismo que se conoce por sus siglas en inglés VOM (VictimOffenderMediation), el cual remite sus primeros antecedentes en 1980 en los EE. UU. Este encuentro se desarrolla por una o variassesiones en conjunto con la víctima y el ofensor, también se observa la participación de una tercera persona quien conduce el proceso y funge como facilitador con el objetivo de solucionar el conflicto y dar respuestas al delito (Zerh, 2007).

Se ha demostrado que en estas sesiones en particular ambos participantes tienen la oportunidad de expresar sus sentimientos cara a cara, así como sus percepciones respecto al ilícito, el objetivo es buscar salidas creativas al crimen con efectos sanadores. Para Howard Zehr es necesario considerar tres aspectos en los procesos restauradores (Zerh, 2007), de primer orden establecer al crimen como consecuencia a la lesión del bien jurídico tutelado, el segundo aspecto refiere la responsabilidad del ofensor en tomar conciencia del daño con la finalidad de repararlo al asumir las consecuencias del delito, y el tercer aspecto puntualiza la reintegración de los participantes.

En este mismo orden de ideas, la ONU reconoce que los encuentros restaurativos favorecen en la disuasión del delito, por lo tanto, se deben considerar en los períodos de la pena privativa de la li- 
bertad en misión de fungir con resultados transformadores (Naciones Unidas, 2015). Por lo tanto, se busca empoderar a la víctima para efectos de enmendar el daño causado por el agresor con el reconocimiento que subyace al activo de reparar el daño en el ámbito moral y material, desde este contexto el infractor puede dimensionar el impacto que ha causado la conducta antijurídica lo cual le ayuda a visionar un comportamiento benéfico en el futuro.

Estos encuentros restaurativos son la fórmula para que la víctima sea escuchada y la oportunidad de participar directamente en el proceso, la realidad es que una vez que integra un proceso penal se generan inquietudes de desconfianza, sin olvidar que acontecen sentimientos de inseguridad en cuanto se ha violentado el bien jurídico tutelado, en función de los encuentros restaurativos la víctima adquiere voz en el proceso toda vez que su reunión es cara a cara con el ofensor (Alatorre, Valdez y Novoa, 2014).

Por ello se debe considerar la aplicación de Leyes sustantivas como la Ley General de Víctimas en la República Mexicana, como se ha venido comentando líneas anteriores se caracteriza por visualizar al derecho con cualidades humanísticas, en ella se sustenta las necesidades de la víctima conforme a lo establecido en la reparación del daño de una manera integral, además faculta al Estado para enmendar los daños de forma subsidiaria, es decir, en el supuesto que las circunstancias o los medios no favorecieran al activo para responder ante el evento el Estado tiene la obligación de dar respuesta en este sentido, por lo tanto, dicho ordenamiento legal garantiza la aplicación de la justicia restaurativa.

\section{Conferencias restaurativas}

Los primeros antecedentes con enfoque alternativo de justicia restaurativa se remontan en Canadá hacia los primeros años de 1970, de tal forma, las prácticas restauradoras han venido evolucionando en los últimos años. En este con- texto, se pueden mencionar los programas reconciliadores que integran el encuentro cara a cara víctima ofensor; las conferencias restaurativas mismas que se constituyen en conferencias de grupos familiares y conferencias comunitarias y los círculos restauradores que a su vez integran círculos de paz o círculos de sentencia.

Dicha metodología restauradora ha sido utilizada en el desarrollo de medidas alternativas en el sistema de justicia penal, en específico las conferencias de grupos familiares se pueden encontrar en Nueva Zelanda ante el compendio normativo de 1986 conocido como Proyecto de Ley sobre Niños y Jóvenes en Nueva Zelanda (McCold, 2013), en este modelo se integra a todas las personas interesadas en la protección de jóvenes ofensores así como la participación de los padres o miembros de la familia con la finalidad de llegar a un consenso y establecer soluciones en la reparación del daño.

A través de las conferencias restaurativas se reconoce la oportunidad que tiene la víctima de narrar su experiencia, así como expresar el impacto que le ha originado la comisión del delito y las marcas que ha dejado en su vida, se observa la participación de diversas redes de apoyo se pueden incluir, profesionales, defensores, amistades, así como miembros de la familia de la víctima y del ofensor, estos grupos son guiados por el facilitador con la intención de abordar el delito, reestructurar el tejido social y disuadir la conducta anti social.

Las conferencias familiares es caracterizada por la participación de los miembros de la familia en el conflicto penal, es decir, la familia auxilia en el contexto emocional y a su vez coadyuva con efectos colaborativos en el proceso de responsabilización del delito (Merino, Romera, \& Carlos, 1998), de tal forma que una vez llegado al consenso el núcleo familiar funge como apoyo en el infractor de la norma para cumplir en los términos acordados. 
Así mismo en las conferencias de grupos familiares prepondera la teoría de la vergüenza reintegrativa interpuesta por John Braithwaite, dicha teoría actúa en retraimiento para cambio en el comportamiento del ofensor, toda vez que se originan sentimientos de culpabilidad ante la presencia de sus familiares quienes de forma colateral demuestran su apoyo y comprensión (Braithwaite, 2002) para la solución del conflicto penal, permitiendo un cambio positivo en el ofensor evitando la reincidencia del delito.

Por su parte, el Instituto Internacional de Prácticas Restaurativas o International Institute for Restorative Practices (conocido por las siglas en inglés IIRP), reconoce la importancia de la intervención de los familiares en virtud de fortalecer los vínculos entre los participantes, mejorando la conducta humana (Costello, Wachtel, \& Wachtel, 2011), en aplicación a los principios fundamentales de la no violencia mediante los efectos que origina los procesos restaurativos con argumento a los valores de la solución del conflicto de forma pacífica, se ha visto que en el caso de conflictos escolares reduce la violencia y el bullying.

Algunos autores manifiestan diversas fases a seguir en estos procesos restaurativos (Merino, Romera, \& Carlos, 1998): en la primera fase se deben valorar las circunstancias de cada familia para integrarse en el proceso; la segunda fase consta de la preparación de cada uno de los participantes; tercera fase concierne en la exposición de los puntos de vista de los intervinientes con miras a desarrollar un plan de reparación; cuarta fase consiste en presentar de manera formal a las autoridades el instrumento jurídico; siguiendo este orden de ideas, es necesario agregar una etapa final consistente en dar un seguimiento cuyo objetivo es conocer el estatus del cumplimiento del acuerdo.

Respecto las conferencias comunitarias, éstas se integran por foros en la sociedad de interacciones cotidianas y se utiliza en varios contex- tos no delictivos, es decir, se busca visualizar la transgresión a la norma en el ámbito escolar, universitario, laboral y social (McCold, 2013), con ello prevenir conductas criminales a través de los principios de la justicia restaurativa.

Dichas prácticas restaurativas suscitan la expresión de emociones mediante un diálogo directo entre los particulares ante el reclamo de la atención de los intereses de la víctima, en colaboración de las redes de apoyo para desarrollar el plan de reparación en solución a la controversia penal y de manera posterior vigilar el comportamiento del ofensor a manera de asegurar el cumplimiento del acuerdo.

\section{Los Círculos de Paz}

La aplicación de los Círculos restaurativos integra la participación de la víctima y el ofensor, también se pueden involucrar miembros de la familia, abogados, defensores, especialistas en la materia, trabajadores sociales, policía, jueces, ministerio público, directivos académicos y cualquier otra persona allegada a la víctima y victimario, en este sentido, se considera como una dinámica flexible para desarrollar estrategias de reintegración, a través de perspectivas profesionales utilizando como herramienta principal el diálogo con la finalidad de elaborar un plan de satisfacción para las víctimas

En este apartado se logra diferenciar los objetivos que establecen los Círculos de Pazy los Círculos de Sentencia. La informalidad de reunir a los participantes y agruparlos en Círculos de Paz fortalece la relación interpersonal y origina un ambiente de confianza entre los intervinientes (Costello, Wachtel, \& Wachtel, 2011). Al estar organizados para poder verse y oírse en forma equidistante, se pueden buscar concretamente soluciones de interés común, y con ello planes de acción, en mejora de transmitir los conocimientos entre los mismos participantes, porque se encuentran en un 
plano de igualdad y confianza (Martínez, 2017).

En cuanto a las metas que persigue un Círculo de Paz incluye promover el restablecimiento de todas las partes afectadas, así como otorga la oportunidad al infractor de enmendar el daño causado y se motiva a la familia del ofensor, amigos y comunidad a participar en el círculo para encontrar resoluciones constructivas, dirigiendo causas fundamentales de conducta criminal, construyendo un sentido de comunidad alrededor de los valores compartidos de la sociedad (Domingo, 2008).

Se deja de manifiesto que el círculo de paz, va más allá de reunir a la víctima y el imputado ya que involucra a la comunidad en el proceso para la toma de las mejores decisiones (Umbreit \& Cores, 2006), estos procesos también integran la participación de un facilitador ante la postura de ser imparcial, sin embargo se observa que carece de neutralidad ya que funge como otra persona más en el proceso restaurativo en cuanto tiene la oportunidad de expresar sus experiencias y relatar sus sentimientos (Alatorre, Valdez y Novoa, 2014).

Como todo sistema debidamente organizado, se requiere de una metodología y en lo particular, se cuenta con una que señala cuatro fases que van desde la valoración, hasta la aplicación del acuerdo (Choya, 2014):

1) Valoración. Incluye valorar la situación para contemplar la posibilidad de ser partícipe en el Círculo de Paz.

2) Preparación. En esta fase se determina quienes serán los participantes, seguidamente se explican los pasos a seguir, dando toda la información a sus participantes.

3) Círculo. El círculo se lleva a cabo por un facilitador, quien invita a los participantes a sentarse de manera redonda. En esta fase se observan cuatro lineamientos a seguir: la presentación, la construcción de la confianza, la identificación del problema y el desarrollo del plan de acción.

4) Seguimiento: esta fase aplica para valorar la aplicación del acuerdo.

Ahora bien, el Círculo de Sentencias generalmente es solicitado por el victimario, sin embargo, no excluye a la víctima ni al órgano jurisdiccional. Una vez que se ha llegado al consenso se presenta al juez en la modalidad de propuesta de sentencia, previa aceptación de la responsabilidad por parte del agresor, la voluntad de rehabilitarse, así como el proceso a seguir en la rehabilitación (Choya, 2014).

Se ha demostrado que los efectos de los Círculos restauradores generan herramienta de diálogos comunicativos mientras se pasan un objeto para efectos de ordenar el turno de hablar entre los intervinientes, lo cual genera equilibrio entre ellos mismos y una conducta reflexiva ante el conflicto penal, por lo tanto busca respuestas positivas y por ende transformadoras en la comisión del delito.

\section{Cultura de Paz}

Cuando hablamos de construir sociedades pacíficas, nos encontramos ante la necesidad de abordar la problemática del conflicto, violencia y guerra, dichos conceptos son de materia en aplicación en los MASC (Gorjón \& Sáenz, 2010). Es importante tomar en consideración las prácticas restaurativas que se han venido desarrollando en los países impulsores de la justicia restaurativa como Estados Unidos, Nueva Zelanda y Canadá, a través de ellos se ha demostrado que los modelos restauradores transforman la comisión del delito con efectos sanadores, en base a lo anterior se debe considerar un país subsidiario en materia de justicia restaurativa y por ende favorecedor en la Paz de las sociedades (Rivas, 2016).

En este sentido, debemos partir de la idea de 
transformar los conflictos para contribuir a la Paz Social, en cuanto el conflicto deviene como resultado de un enfrentamiento de intereses entre dos o más personas, donde se trata de romper la resistencia del otro usando generalmente algún tipo de violencia, en el ámbito penal, se genera el delito, lo que puede y ha resultado en consecuencias graves, incluso podría llevar al aniquilamiento físico de la otra persona (Marquez, 2012).

A partir de la mencionada reforma del 2008, se ha evidenciado un cambio en un nuevo diseño de las políticas públicas del sistema de justicia penal, cuyo objetivo es la reparación del daño a través de herramientas que fomenten la cultura del dialogo, la justicia y la equidad.

En este sentido con la reforma se otorga una mayor certeza jurídica a las víctimas y a los presuntos responsables, así mismo se crean los mecanismos alternativos de solución de controversias (Díaz, 2013). Con ello la aplicación de la justicia restaurativa como un nuevo paradigma que tiende a fortalecer la aplicación de justicia en el Estado Mexicano.

Los mecanismos alternativos favorecen en la incrementación de los valores de paz, ya que contribuyen a una justicia penal no inquisitiva, con el objetivo de que las partes resuelvan por ellos mismos sus conflictos bajo un esquema positivo y sanador, con una franca tendencia a la cultura de la paz.

\section{Resultados}

En el desarrollo de este artículo, se observa un nuevo enfoque de justicia, fungiendo como un parteaguas en la política criminal, en virtud de visualizar un cambio social con apuntalamiento a los criterios de universalidad y equidad en todos y cada uno de los procesos restaurativos, cuyos principios hacen de lado el sistema tradicional de justicia. De tal manera, apuntala un proceso de transición en este nuevo paradigma de justicia restaurativa que atribuye al Estado Mexicano.

Vistas las cosas desde este ángulo, se logra demostrar que la Justicia Restaurativa integra diversosfactoresfundamentales en la resolución de conflictos y la aplicación de mecanismos alternativos para lograr entender que las formas tradicionales y originarias no han permitido una fluidez en la incorporación de enfoques restaurativos en México, de tal manera que el juzgamiento del transgresor con una sentencia condenatoria no satisface por completo las necesidades de las víctimas, incluso una vez en prisión, la disciplina punitiva no genera cambios auténticos en el comportamiento del ofensor.

En este mismo contexto, los encuentros restaurativos, las conferencias de grupos y los círculos de paz, fungen como herramientas en la Justicia Restaurativa y proponen a la víctima en el protagonismo del conflicto penal tomando en consideración en todo momento procesal al infractor de la norma, pues en conjunto se establece un cúmulo de ideasy necesidades para solucionar la controversia y a su vez recomponer el tejido social que se ha fracturado.

De tal manera, los resultados de la Justicia Restaurativa ponen en evidencia los sentimientos y las reflexiones que los participantes desarrollan en el evento con un efecto transformador, ya que a través de las descargas emocionales los integrantes muestran por primera vez su lado humano, así mismo se constituye un panorama armonioso en las sociedades, versus lo caracterizado por el iuspuniendifacultativodel Estadoyconocidopor el sistema inquisitivo que meramente visualiza el castigo y estigmatiza a los participantes.

\section{Conclusiones}

La inserción de los Mecanismos Alternativos para la Solución de los Controversias en la administración, procuración de justicia y 
ejecución de penas privativas de libertad del Estado Mexicano, ha traído como consecuencia un crecimiento en el ámbito jurídico, social y cultural, pues la aplicación de la mediación penal, conciliación y justicia restaurativa implica un cambio en la sociedad a nivel cultural mediante el fomento de los valores y la cultura del diálogo para que con la participación de los integrantes del conflicto se logre generar una convivencia pacífica y el respeto entre los miembros de la comunidad.

Aunque se ha fortalecido el ordenamiento jurídico mediante el incremento de Leyes que sustentan las medidas alternativas en los conflictos penales, podemos entender que la justicia restaurativa en México se le puede catalogar como un incipiente movimiento social, cuyos objetivos son la restructuración del tejido social, en ella se considera el delito como un daño contra una persona o diversos integrantes de la sociedad, se observa como protagonistas a la víctima y el ofensor en un amplio entendimiento en la controversia penal.

Bajo este contexto se observan grandes retos, el primero de ellos consta en culturizar a la población mexicana sobre el desarrollo de la justicia restaurativa, se debe proyectar la implementación de los programas restaurativos focalizados en el delito y por lo tanto en la población penitenciaria; segundo, es necesario considerar hacer partícipes a todos los actores sociales en los procesos de reconciliación.

A través de la Justicia Restaurativa se asumen de manera consensuada diversas opciones de solución, en cambio en diversas latitudes, encontraremos que hay una madurez en el sentido de la debida aplicación de la justicia restaurativa y pudiésemos considerar empatar algunos preceptos.

En atención al análisis de las Leyes que nos permitimos ponderar, encontramos la imperiosa necesidad de ampliar el tema referente a la Justicia Restaurativa, donde podamos distinguir los diferentes programas y metodologías fundamentales para su aplicación, ya que si bien es cierto, el corpus iuris actual nos habla del deber ser, pero no del cómo hacer, amén de que todo el esquema jurídico nacional referente al sistema de aplicación de justicia oral fortalece la transparencia en el proceso y protege los derechos humanos con afinidad al derecho penal.

Para que el Estado Mexicano garantice el debido proceso de la metodología restaurativa y a su vez coadyuve en la disuasión del delito resulta imprescindible:

a) Fortalecer los vínculos sociales entre la comunidad, población penitenciaria y Estado.

b) Concientizar a la sociedad en la cultura diálogo y el fomento de la cultura de Paz a través de valores que rechacen la violencia

c) Robustecer el principio resocializador a través de diseños con estrategias de justicia restaurativas.

d) Instrumentalizar Políticas Públicas enfocadas en el desarrollo de estrategias de justicia restaurativas.

Es importante considerar que los efectos a posteriori de una participación en procesos restaurativos originan beneficios sociales, individuales, familiares, colaborativos e integrativos de valores argumentativos en la No-violencia, con la finalidad de re-orientar las normas punitivas a un ordenamiento legal de carácter humanístico con efectos resocializadores. 


\section{Referencias}

Ahumada, M. d. (2011). La conciliación: un medio de justicia restaurativa, análisis y reflexiones de su implementacion en el delito de inasistencia alimenticia en Colombia. Revista Facultad de Derecho y Ciencias Políticas, 41(114), 11-40.

Alatorre, F., Valdez, A. \& Novoa, J. (2014). Confianza es destino: Un análisis de la percepción de los electores universitarios en la elección presidencial de México, 2012. Pensamiento Americano, 7(13), 183-210.

Arboleda López, A. P., Gárces Giraldo, L. F., Murillo Bocanegra, J. E., \& Pineda Carreño, M. (2017). Principio, habilidades y virtudes para el conciliador en derecho. Revista pensamiento Americano, X(18), 191-200.

Barnett, R. (1977). Restitution: A New Paradigm of Criminal Justice. Recuperado el 28 de 07 de 2016, de scholarship.law.georgetown.edu/cgi/viewcontent. cgi?article...

Blanco Suárez, R. (2014). Los problemas de las reformas al proceso penal. Una necesaria visión desde lo multidisciplinario. Revista Justicia(25), 106-13.

Braithwaite, J. (2002). Restorative Justice and Responsive Regulation. New York: Oxford.

Cabello, P. (2015). La multidisciplinariedad de la mediación y sus ámbitos de aplicación. México: Tirant Lo Blanch.

Cámara de Diputados del H. Congreso de la Unión. (29 de Diciembre de 2014). Ley Nacional de Mecanismos Alternativos de Solución de Controversias en Materia Penal. Ley Nacional de Mecanismos Alternativos de Solución de Controversias en Materia Penal. México, México, México: Cámara de Diputados del H. Congreso de la Unión.

Cámara de Diputados del H. Congreso de la Unión. (16 de Junio de 2016). Ley Nacional de Ejecución Penal. Ley Nacional de Ejecución Penal. México, México, México: Cámara de Diputados del H. Congreso de la Unión.

Cárcamo Vásquez, H. (2005). Hermenéutica y Análisis Cualitativo. Revista de Epistemología de Ciencias Sociales, 204-216.

Carnelutti, F. (1982). La Prueba Civil. Buenos Aires, Argentina: Ediciones Desalma.

Carrillo Pumarejo, R. E., \& Álvarez Martínez, F. (2012). El crimen: una perspectiva desde los derechos humanos. Revista pensamiento Americano, V(9), 37-43.

Choya, N. (2014). Prácticas restaurativas: círculos y conferencias. País Vasco.
Costello, B., Wachtel, J., \& Wachtel, T. (2011). Manual de Prácticas Restaurativas. Pennsylvania, USA: International Institute -For Restorative Practices.

Diario Oficial de la Federación. (19 de Septiembre de 2017). Diario Oficial de la Federación. Obtenido de Leyes y Reglamentos: www.dof.gob.mx/

Díaz, I. (2013). La Mediación en el Sistema de Justicia Penal: Justicia Restaurativa en México y España. México: Comisión Editorial del Instituto de Investigaciones Jurídicas.

Domingo, V. (2008). Justicia Restaurativa y Mediación Penal. Revista de Derecho Penal. Lex Nova(23). Obtenido de www.justiciarestaurativa.org/news/ justicia-restaurativa-y-mediacion-penal

Eiras, U. (2005). Mediación penal de la práctica a la teoría. Buenos Aires, Argentina: Histórica.

Galain, P. (2009). Mediación penal como forma alternativa de resolución de conflictos: la construccion de un sistema penal sin jueces. Revista Penal Num. 24, 7189.

García, S. (2014). Comentarios sobre el Codigo Nacional de Procediemientos Penales del 2014. Boletín Mexicano de Derecho Comparado, Nueva Serie, Número 141, $1186-1187$.

Gorjón Gómez, G. d., Cabello Tijerina, P. A., Gorjón Gómez, F. J., Saénz López, S. C., Sánchez García, A., Steele Garza, J. G., ... Zaragoza Huerta, J. (2015). Comentarios a la Ley Nacional de Mecanismos Alternativos de Solución de Controversias en Materia Penal. México, D. F.: Tirant Lo Blanch.

Gorjón, F. (2013). El Valor Intangible de los MASC. Revista Nuevo Sistema de Justicia Penal, No. VI, SETEC, México, 48.

Gorjón, F., \& Sáenz, K. (2010). Métodos Alternos de Solución de Controversias: Enfoque Educativo por Competencias. México: Grupo Editorial Patria.

Gorjón, F., \& Steele, J. (2008). Metodos Alternativos de Solución de Conflictos. México: Oxford.

Hernández Sampieri, R., Fernández Collado, C., \& Baptista Lucio, P. (2006). Metodologia de la Investigación (Cuarta ed.). Mexico: McGraw-Gil.

Islas Colín, A., Vera H., D., \& Miranda-Medina, C. (2018). La cultura de paz en las políticas de Educación Superior de México, Colombia y Ecuador. Revista Educación y Humanismo, 20(32), 279-291.

Marquez, A. (2012). La Mediación como Mecanismo de Justicia Restaurativa. Revista Prolegómenos, 149-171.

Pensamiento Americano Vol. 11 - No. 21 • Julio-Diciembre • Corporación Universitaria Americana • Barranquilla, Colombia • ISSN: 2027-2448 · http://coruniamericana.edu.co/publicaciones/ojs/index.php/pensamientoamericano 
Martínez, P. Y. (Junio de 2017). Tesis doctoral. El Círculo de Paz como herramienta de justicia restaurativa para la reinserción social en el sistema penitenciario mexicano. Caso centro de reinserción social Cadereyta. Monterrey, Nuevo León, México: Universidad Autónoma de Nuevo León.

McCold, P. (2013). The recent history of restorative justice. Mediation, circles and conferencing. Delito $y$ Sociedad, 22(36), 9-44.

Merino, C., Romera, \& Carlos. (1998). Conferencias de grupos familiares y sentencias circulares: dos formas ancestrales de resolucion de conflictos dentro del paradigma restaurativo. Eguskilore.(12), 285-303.

Mosquera Rentería, J. (2015). Derechos de la minorías sexuales: retos contemporáneos de la resocialización. Revista Justicia, 20(28), 121-136.

Naciones Unidas. (2015). Programas y Actividades del desarrollo de las Naciones. Obtenido de Naciones Unidas: www.un.org/es/globalissues/development/ agencies.shtml

Neuman, E. (2005). La Mediación Penal y la Justicia Restaurativa. Argentina: Porrúa.

Olson, C. (2001). justicia Penal y sobrepoblación Penitenciaria. Costa Rica: Siglo XXI Editores S. A. de C. V.

Organización de las Naciones Unidas. (2006). Declaración sobre los Principios Fundamentales de Justicia para las víctimas del delito y el abuso del poder. Recuperado el 2016 de Agosto de 04, de Recopilación de reglas y normas de las Naciones Unidas en la esfera de la prevención del delito y la justicia penal: https://www.unodc.org/pdf/compendium/ compendium_2006_es_part_03_02.pdf

Organización de las Naciones Unidas. (2006). Manual sobre programas de Justicia Restaurativa. Recuperado el 16 de Abril de 2015, de Manual sobre programas de Justicia Restaurativa.: https://www. unodc.org/...and.../Manual_sobre_programas_de_ justicia_restaurativa.pd...

Organización Naciones Unidas. (2014). Instituto Latinoamericano de las Naciones Unidas para la Prevención del Delito y el Tratamiento del Delincuente. Recuperado el Septiembre de 2015, de http://www.ilanud.or.cr/programas/medidasrelativas-a-la-justicia-restitutiva.html

Rivas, J. (2016). Liderar la guerra, liderar la paz. Estilos de liderazgo en Colombia (1982-2014). Pensamiento Americano, 9(16), 27-44.

Rousset, A. (2011). El Concepto de Repa ración Integral en la Jurisprudencia de la Corte Interamericana de los Derechos Humanos. Revista Internacional de Derechos Humanos. ISNN 2250-5210, 59-79.

Suprema Corte de Justicia de la Nacion. (Marzo de 2006). Semanario Judicial de la Federación y su Gaceta. Recuperado el 04 de Agosto de 2016, de Reparación del daño: sjf.scjn.gob.mx/sjfsist/Documentos/ Tesis/175/175459.pdf

Umbreit, \& Cores. (2006). Resturative Justice Dialogue: Eidence- Based practiced School of Social Work. cololege of Economy.

Zerh, H. (2007). El Pequeño Libro de la Justicia Restaurativa. E. U. A: Good Books.
2018, Vol. 11(21) 79-95 (CThe Author(s) 2018 Reprints and permission: www.americana.edu.co 